\title{
Activity ex vivo of cytotoxic drugs in patient samples of peritoneal carcinomatosis with special focus on colorectal cancer
}

\author{
Peter H Cashin 1*, Haile Mahteme ${ }^{1}$, Wilhelm Graf ${ }^{1}$, Henning Karlsson ${ }^{2}$, Rolf Larsson ${ }^{2}$ and Peter Nygren ${ }^{3}$
}

\begin{abstract}
Background: The optimal choice of cytotoxic drugs for intraperitoneal chemotherapy (IPC) in conjunction with cytoreductive surgery (CRS) for treatment of peritoneal carcinomatosis (PC) is poorly defined. We investigated drug sensitivity ex vivo in patient samples of various PC tumor types and correlated clinical outcome to drug sensitivity within the subset of PC from colorectal cancer (CRC).

Methods: PC tissue samples $(n=174)$ from mesothelioma, pseudomyxoma peritonei (PMP), ovarian cancer, CRC or appendix cancer were analyzed ex vivo for sensitivity to oxaliplatin, cisplatin, mitomycin C, melphalan, irinotecan, docetaxel, doxorubicin and 5-FU. Clinicopathological variables and outcome data were collected for the CRC subset.

Results: Mesothelioma and ovarian cancer were generally more drug sensitive than CRC, appendix cancer and PMP. Oxaliplatin showed the most favorable ratio between achievable IPC concentration and ex vivo drug sensitivity. Drug sensitivity in CRC varied considerably between individual samples. Ex vivo drug sensitivity did not obviously correlate to time-to-progression (TTP) in individual patients.

Conclusions: Drug-sensitivity varies considerably between PC diagnoses and individual patients arguing for individualized therapy in IPC rather than standard diagnosis-specific therapy. However, in the current paradigm of treatment according to diagnosis, oxaliplatin is seemingly the preferred drug for IPC from a drug sensitivity and concentration perspective. In the CRC subset, analysis of correlation between ex vivo drug sensitivity and TTP was inconclusive due to the heterogeneous nature of the data.
\end{abstract}

Keywords: Chemotherapy resistance, Cytoreductive surgery, Drug sensitivity, Fluorometric microculture cytotoxicity assay, Hyperthermic intraperitoneal chemotherapy, Peritoneal carcinomatosis

\section{Background}

Peritoneal carcinomatosis (PC) was previously regarded as an incurable form of malignant disease with a poor prognosis, and the intention of treatment was palliative. However, aggressive cytoreductive surgery (CRS) followed by intraperitoneal chemotherapy (IPC), may produce prolonged long-term survival and even cure [1-5]. The most recent development in the management of $\mathrm{PC}$ is the intraoperative use of hyperthermic intraperitoneal chemotherapy (HIPEC) [6].

The individual therapeutic impact of cytoreductive surgery and IPC, respectively, has not been sufficiently

\footnotetext{
* Correspondence: peter.cashin@surgsci.uu.se

'Department of Surgical Sciences, Section of Surgery, Akademiska Sjukhuset, Uppsala University, Uppsala S-751 85, Sweden

Full list of author information is available at the end of the article
}

clarified [7]. In ovarian cancer, IPC is more active than the corresponding drug given iv and in gastric cancer, IPC has been shown to add benefit to CRS and systemic chemotherapy $[8,9]$. In PC from colorectal cancer (CRC), the role of IPC when added to CRS has not been elucidated in randomized trials but cytoreductive surgery, IPC, and systemic chemotherapy is favorable compared with systemic chemotherapy and palliative surgery [10]. An experimental study in the rat demonstrated a significant increase in survival when adding IPC to cytoreductive surgery vs. cytoreductive surgery alone [11].

The selection of drugs for IPC has mostly been based on the experience from systemic administration, pharmacodynamic properties, hyperthermic enhancement, technical feasibility, pharmacokinetics and tolerance [12,13]. The IPC 
protocols in use do not take into consideration possible differences in drug sensitivity in the different tumor types or differences in tumor cell sensitivity between individual patients. Currently, cisplatin, doxorubicin, mitomycin C, oxaliplatin and irinotecan, as single drugs or sometimes combined, are the most commonly used drugs for HIPEC treatment [14-18]. A more differential approach to drug selection for the IPC in PC might provide more benefit from this part of the treatment of $\mathrm{PC}$.

With this background, we investigated ex vivo the activity of standard cytotoxic drugs in IPC on tumor cells derived from patients with various types of PC using an ex vivo model reflecting clinical drug activity. The aims were to investigate differences in drug sensitivity between various PC tumor types and individual patient samples and to investigate if differences in clinical outcome are associated with drug sensitivity within the subset of CRC.

\section{Methods}

\section{Tumor sampling and cell preparation}

Tumor sampling of patients with PC from appendix cancer, CRC, pseudomyxoma peritonei (PMP), ovarian cancer, or mesothelioma was performed intraoperatively during cytoreductive surgery prior to IPC. Leukemia sampling was by vein puncture at routine blood sampling and mononuclear cells (MNCs) were prepared from buffy coats from healthy blood donors. Tumour sampling and data collection was based on patient informed consent as approved by the regional ethical committee in Uppsala (Uppsala Etiknämnd: Dnr 2007/237).

Tumor cells from solid tumor tissue were prepared by collagenase digestion as described [19]. Leukemia cells and MNCs were collected by Ficoll-Hypaque (Pharmacia, Uppsala, Sweden) gradient centrifugation [20].

The cells obtained from the solid tumors were single cells or small cell clusters with $\geq 90 \%$ viability and with less than $30 \%$ contaminating non-malignant cells, as judged by morphological examinations of May-GrünwaldGiemsa-stained cytocentrifugate preparations. Approximately $85 \%$ of all samples obtained fulfilled the criteria for a successful assay (see below) and were included in this study. The numbers and types of samples included are detailed in Table 1.

\section{Drugs and measurement of drug sensitivity ex vivo}

The cytotoxic drugs melphalan (Mel; GlaxoSmithKline, Stockholm, Sweden), cisplatin (Cisp; Bristol-Myers Squibb, Stockholm, Sweden), oxaliplatin (Oxali; SanofiSynthelabo, Stockholm, Sweden), doxorubicin (Dox; Pfizer, Stockholm, Sweden), docetaxel (Doce), 5-fluorouracil (5FU; Roche, Stockholm, Sweden), mitomycin C (MitC; BristolMyers Squibb) and irinotecan (Irino; Pfizer) were from commercially available clinical preparations. The drugs were tested at three 10-fold dilutions from the maximal
Table 1 Number of patient samples included in the analyses

\begin{tabular}{llll}
\hline CELL TYPE & TREATED $(\boldsymbol{n})$ & UNTREATED $(\boldsymbol{n})$ & TOTAL $(\boldsymbol{n})$ \\
\hline Pseudomyxoma & 24 & 48 & 72 \\
Mesothelioma & 7 & 8 & 15 \\
Appendix & 11 & 5 & 16 \\
Colorectal & 39 & 13 & 52 \\
Ovarian & 15 & 4 & 19 \\
AML & 6 & 6 & 12 \\
CLL & 0 & 12 & 12 \\
MNC & NA & NA & 44
\end{tabular}

Treated/untreated refers to whether the patients sampled had previously received chemotherapy (treated) or not (untreated).

Abbreviations: $A M L$ acute myeloblastic leukemia, CLL chronic lymphocytic leukemia, MNC mononuclear cells, NA not applicable.

concentration $(\mu \mathrm{M})$ of 100 for Mel, 100 for Cisp, 100 for Oxali, 10 for Dox, 100 for Doce, 1000 for 5-FU, 100 for MitC and 1000 for Irino. Irino shows relevant activity ex vivo under conditions of the FMCA despite being considered as a prodrug [21].

The semi-automated fluorometric microculture cytotoxicity assay (FMCA), described in detail previously, was used to assess drug sensitivity [22]. The method is based on measurement of fluorescence generated from hydrolysis of fluorescein diacetate (FDA) to fluorescein by cells with intact plasma membranes. 384-well microplates (Nunc) were prepared with $5 \mu \mathrm{l}$ drug solution at 10 times the final drug concentration using the pipetting robot BioMek 2000 (Beckman Coulter). The plates were then stored at $-70^{\circ} \mathrm{C}$ until further use.

Tumor cells from patient samples $(5,000$ cells/well for the PC samples and 40,000 cells per well for leukemia and MNCs) in $45 \mu \mathrm{l}$ were seeded in the drug-prepared 384-well plates using the pipetting robot Precision 2000 (Bio-Tek Instruments Inc., Winooski, VT). Three columns without drugs served as controls and one column with medium only served as blank.

The culture plates were then incubated at $37^{\circ} \mathrm{C}$ in humidified atmosphere containing 95\% air and 5\% $\mathrm{CO}_{2}$. After $72 \mathrm{~h}$ incubation, the culture medium was washed away and $50 \mu \mathrm{l} /$ well of a physiological buffer containing $10 \mu \mathrm{g} / \mathrm{ml}$ of the vital dye fluorescein diacetate (FDA) were added to control, experimental and blank wells. After incubation for 30-45 $\mathrm{min}$ at $37^{\circ} \mathrm{C}$, the fluorescence from each well was read in a Fluoroscan 2 (Labsystems OY, Helsinki, Finland).

\section{Quality control and quantification of results}

Quality criteria for a successful assay were: $\geq 70 \%$ tumor cells in the cell preparation prior to incubation and/or on the assay day, a fluorescence signal in control cultures of $\geq$ five times mean blank values, and a coefficient of variation of cell survival in control cultures of $\leq 30 \%$. The results obtained by the viability indicator FDA are 
presented as survival index (SI), defined as the fluorescence of the test expressed as a percentage of control cultures, with blank values subtracted.

Concentration-response SI data were used to calculate the $50 \%$ inhibitory concentrations, i.e. the drug concentration producing a SI of $50 \%,\left(\mathrm{IC}_{50}\right)$. This was done using non-linear regression to a standard sigmoidal dose-response model in GraphPad Prism version 5 for Mac (GraphPad Software, San Diego, CA, USA). Data are presented as mean values \pm SE for the number of experiments/samples indicated.

\section{Subgroup analysis of patients with peritoneal carcinomatosis of colorectal origin}

Histopathological data concerning lymph node positive status, tumor grading, mucinous status, and signet cells were collected from the pathological report of the primary tumor resection. Date and cause of death, time to disease progression, completeness of cytoreduction score (CC), systemic chemotherapy prior to cytoreductive surgery and use of adjuvant systemic chemotherapy after CRS and IPC were also collected. Time to progression (TTP) was selected as the clinically most relevant outcome measure when investigating the correlation between ex vivo drug sensitivity and clinical outcome.

Out of the 52 patients with PC from CRC, fifteen did not receive IPC due to too advanced disease. The IPC in the remaining 37 patients was intraoperative hyperthermic IPC (HIPEC) or sequential postoperative intraperitoneal chemotherapy (SPIC). HIPEC consisted of oxaliplatin (360-460 $\left.\mathrm{mg} / \mathrm{m}^{2}\right)$ administered intraperitoneally (ip) with 5-FU (400-500 $\mathrm{mg} / \mathrm{m}^{2}$ plus folic acid) intravenously (iv) in 12 patients. HIPEC with irinotecan and oxaliplatin $\left(360 \mathrm{mg} / \mathrm{m}^{2}\right.$ of each drug) with 5 -FU and folic acid iv was used in 18 patients. SPIC with 5 -FU $\left(500 \mathrm{mg} / \mathrm{m}^{2}\right)$ infusion ip through a PORT á CATH (No. 21-2000-04, SIMS Deltec, Inc., St Paul, MN, USA) at 4-6 week intervals for 6 months postoperatively was used in 7 patients. For systemic chemotherapy prior to the IPC $(n=39)$, the interval between last administration and surgery had to be at least 4 weeks. Adjuvant chemotherapy was administered in 15 patients and the most common regimen was oxaliplatin and 5 - $\mathrm{FU}(\mathrm{n}=8)$.

There were 27 patients with a CC score of 0 receiving IPC treatment and these were considered suitable for assessment of the ex vivo - in vivo correlation. There was missing data on recurrences in 4 patients and so the analysis was possible in 23 patients. In line with previous principles for correlation of ex vivo drug sensitivity to clinical outcome [19], patients were scored as ex vivo 'sensitive' if they were treated with at least one drug with $\mathrm{IC}_{50}$ value below the median for the whole study cohort and as ex vivo 'resistant" if they had received only drugs with all $\mathrm{IC}_{50}$ values above the median.

\section{Statistics}

Statistical inferences between several means were performed by one-way ANOVA with Tukey's multiple comparison post-test of group means or, for comparison of two means, by Student's t-test. Cross-resistance between selected drugs was analyzed by Spearman rank correlation. The slope of the regression line was calculated with the least squares method. Time to progression was compared using the Kaplan-Meier curve's median survival and the log-rank test. For the CRC subgroup, the prognostic importance of clinicopathological variables and ex vivo drug sensitivity for TTP was assessed in a Cox regression model. All univariate results with $\mathrm{p}<0.05$ were included in the multivariable analysis using an all-effects function of both forward and backward stepwise analysis. The level of significance for all statistical tests was set to $\mathrm{p}<0.05$. The statistics software used in these analyses was STATISTICA 10.1 (StatSoft Inc, Tulsa, OK, USA).

\section{Results}

\section{Patient samples}

In total, 174 tumor samples from patients operated on for PC, as detailed in Table 1, fulfilled the quality criteria and were analyzed for drug sensitivity. The majority of patients had PMP or CRC and the majority of samples were from patients previously treated with chemotherapy. The clinical characteristics for the CRC patient subgroup are detailed in Table 2 .

\section{Drug sensitivity ex vivo}

Samples from CRC, appendix cancer, and PMP generally had higher $\mathrm{IC}_{50}$ values compared to the other groups with the exception of 5-FU (Figure 1). Mesothelioma samples were surprisingly sensitive across the panel of drugs similarly to ovarian cancer. As expected the leukemia samples were mostly more sensitive or as sensitive as the mesothelioma and ovarian cancer samples.

Based on the $\mathrm{IC}_{50}$ values and the ip concentrations of each drug reached during IPC, as reported in the literature, the ratios between the ip concentrations and the $\mathrm{IC}_{50}$ values were calculated for each drug and PC diagnosis (Table 3). High ratios would in theory be most beneficial. Across all diagnoses, the clearly most beneficial ratio was observed for oxaliplatin.

Some samples were essentially unaffected by the highest drug concentrations tested whereas tumor cells from other samples showed decreased viability even at the lowest concentration tested (Figure 2). The cross-resistance between the different standard drugs investigated was modest to high (Figure 3). In general this means that resistance to one drug would also imply resistance to other drugs. On the other hand, there are clearly many individual samples being resistant to one but sensitive to the other drug, theoretically supporting an individual choice of drugs for the IPC. 
Table 2 Clinical characteristics of the CRC subgroup of 52 patients

\begin{tabular}{|c|c|}
\hline $\begin{array}{l}\text { Characteristics ( } n \text {, except for age } \\
\text { in years and } \mathrm{PCl} \text { in score units) }\end{array}$ & Colorectal cancer \\
\hline Age - mean years $(95 \% \mathrm{Cl})$ & $55(51-59)$ \\
\hline Gender - Male/Female & $21 / 31$ \\
\hline IPC administered & 37 \\
\hline SPIC & 7 \\
\hline HIPEC & 30 \\
\hline Oxaliplatin & 12 \\
\hline Oxaliplatin + irinotecan & 18 \\
\hline $\mathrm{PCl}$ - mean $(95 \% \mathrm{Cl})$ & $21(18-24)$ \\
\hline \multicolumn{2}{|l|}{ CC score } \\
\hline 0 & 27 \\
\hline 1 & 5 \\
\hline 2 & 4 \\
\hline 3 & 16 \\
\hline \multicolumn{2}{|l|}{ Diagnosis } \\
\hline Colon & 47 \\
\hline Rectum & 5 \\
\hline Preoperative chemo & 39 \\
\hline Oxaliplatin/5-FU \pm bev & 30 \\
\hline Oxaliplatin/5-FU + cet & 1 \\
\hline Oxaliplatin/capecitabine \pm bev & 4 \\
\hline Irinotecan/5-FU + bev & 1 \\
\hline Capecitabine & 1 \\
\hline 5-FU alone & 2 \\
\hline Adjuvant systemic chemo & 15 \\
\hline Oxaliplatin/5-FU \pm bev & 8 \\
\hline Irinotecan/5-FU \pm bev & 2 \\
\hline Capecitabine & 1 \\
\hline 5-FU alone & 1 \\
\hline Missing data on drug & 3 \\
\hline
\end{tabular}

Abbreviations: $C R C$ colorectal cancer, $P C I$ peritoneal cancer index, IPC intraperitoneal chemotherapy, SPIC sequential postoperative intraperitoneal chemotherapy, HIPEC hyperthermic intraperitoneal chemotherapy, CC completeness of cytoreduction, Chemo chemotherapy, Bev bevacizumab, Cet cetuximab.

Ex vivo drug sensitivity versus clinicopathological factors for the colorectal cancer subgroup

Table 4 details the mean $\mathrm{IC}_{50}$ values of each drug according to different histopathological categories in the CRC subgroup. The mucinous tumors generally had higher $\mathrm{IC}_{50}$ values compared to those non-mucinous and this was statistically significant or nearly significant for cisplatin (30 vs. $14 \mu \mathrm{M}, \mathrm{p}=0.05$ ) and irinotecan (184 vs. $81 \mu \mathrm{M}$, $\mathrm{p}=0.07)$. Lymph node status at initial diagnosis, tumor grade or signet ring cell type cancer did not consistently affect drug sensitivity. There were essentially no differences in drug sensitivity between CRC PC samples from patients previously exposed to cytotoxic drugs and those being treatment naïve (Table 4).

Analysis of prognostic impact from histopathology variables and drug sensitivity according to a Kaplan-Meier survival analysis and a multivariable Cox regression model are detailed in Table 5. After adjustment for various prognostic factors in a multivariable model, sensitivity to doxorubicin, synchronous PC and macroscopically radical surgery were independently associated with longer TTP.

The results of the analysis of the direct relationships between drug sensitivity ex vivo and clinical outcome in individual CRC PC patients are detailed in Figure 4. The Kaplan-Meier curves essentially overlap in the beginning and do not differ statistically significantly. However, at three years, there are three disease free patients in the sensitive group and none in the resistant group. Longer follow-up and more patients are needed for a conclusive analysis.

\section{Discussion}

The FMCA analysis has previously been shown to predict drug efficacy in the clinic both on the diagnosis level and on the individual patient level in hematological malignancies and ovarian cancer $[19,20,26]$. Therefore, we expect that the FMCA reports clinically relevant drug sensitivity also in the current investigation. CRC, appendix cancer and PMP were generally more resistant to standard cytotoxic drugs than ovarian cancer and mesothelioma. However, for docetaxel in PMP the $\mathrm{IC}_{50}$ value approached that of leukemia. This is an interesting observation as docetaxel also showed the lowest cross-resistance to other drugs.

Mesothelioma was surprisingly relatively sensitive across all drugs tested. Cisplatin, the most common agent both for systemic treatment and IPC in PC from mesothelioma [27], is a good choice based on our findings. This is also in line with the treatment results of CRS and IPC for mesothelioma, where the median survival ranges 34-96 months with 5 year overall survival ranging 33-59\%, which is better than systemic chemotherapy results with median survival ranging from 9 to 12.5 months [27]. Furthermore, abdominal mesothelioma treated with cytoreductive surgery and IPC appears to have better survival than CRC according to a recent French study which is in line with the FMCA results of greater drug sensitivity [28]. Notably, however, given that the pattern of oxaliplatin activity is very similar to that of cisplatin and the much more favorable concentration ratio for oxaliplatin, use of this drug for IPC in mesothelioma might produce even better results.

Despite having similar drug resistance patterns as CRC, PMP has better survival than both CRC and mesothelioma. The explanation for this is probably that the tumor biology of PMP is more indolent compared with mesothelioma and CRC regardless of the drug resistance pattern [29].

Appendix cancer was similar to CRC in drug sensitivity. Thus, the current practice of treating these two entities 

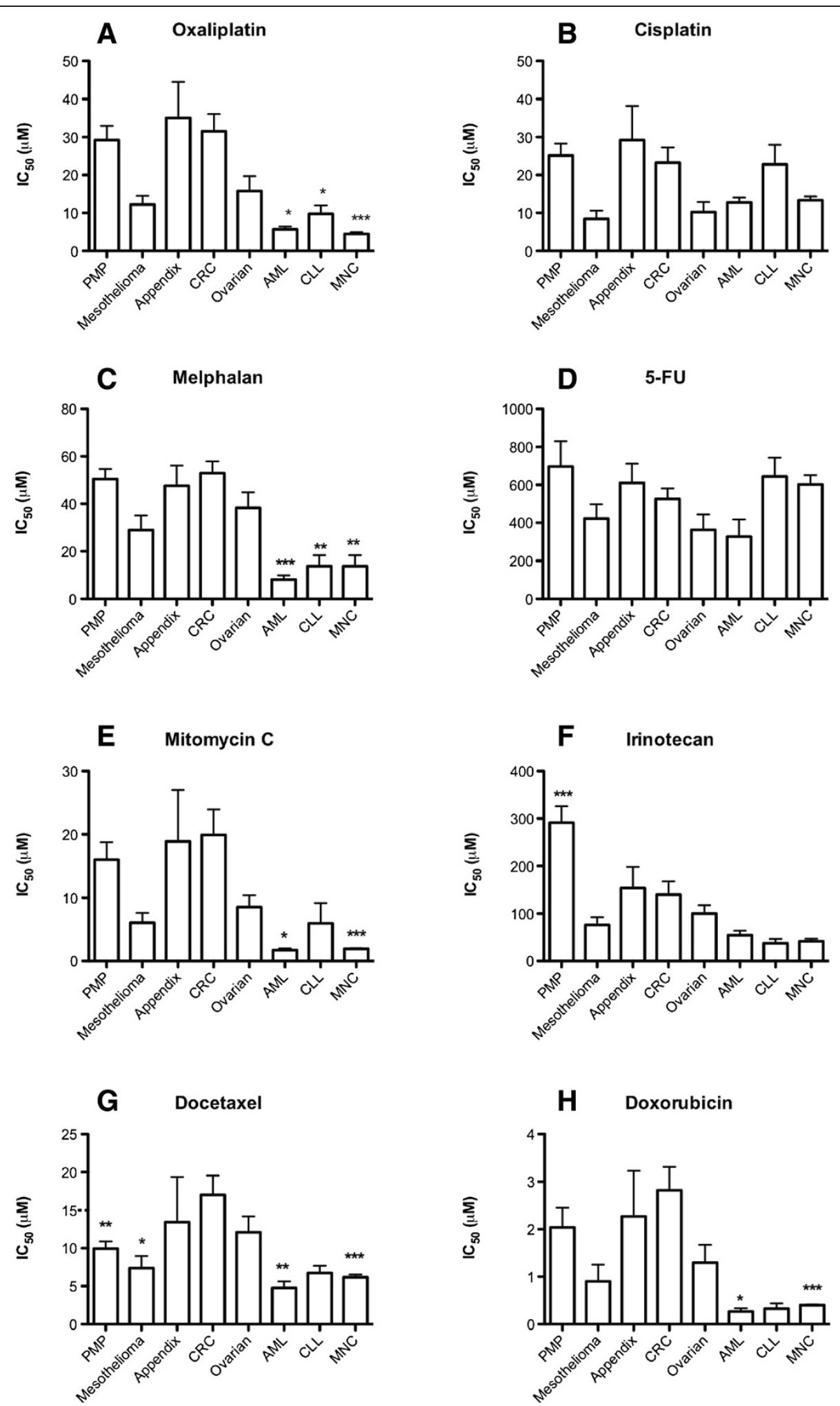

Figure $1 \mathrm{IC}_{50}$ values for standard drugs in all peritoneal carcinoma samples investigated divided for the subtypes indicated. Results are presented as means values + SE. Statistical inference was calculated with 1-way ANOVA with Dunnet's post-test and with the colorectal cancer samples as reference. The following panel of 8 drugs were investigated: A - Oxaliplatin, B - Cisplatin, C - Melphalan, D - 5FU, E - Mitomycin C, $\mathbf{F}$ - Irinotecan, $\mathbf{G}$ - Docetaxel, $\mathbf{H}$ - Doxorubicin. ${ }^{*},{ }^{* *}$ and ${ }^{* * *}$ denotes $P<0.05,0.001$ and 0.0001 vs. colorectal samples, respectively. Absence of asterixes means that no statistical differences compared to colorectal samples were observed. 
Table 3 Concentration ratios between IPC $C_{\max }$ and ex vivo IC $C_{50}$ values

\begin{tabular}{|c|c|c|c|c|c|c|c|c|c|c|}
\hline $\mathrm{C}_{\max }{ }^{\dagger}$ & $\begin{array}{l}I_{50} \\
\text { CRC } \\
\mu M\end{array}$ & $\begin{array}{l}C_{\max } \\
/ I C_{50} \\
C R C\end{array}$ & $\begin{array}{l}\mathrm{IC}_{50} \\
\text { Meso } \\
\mu \mathrm{M}\end{array}$ & $\begin{array}{l}C_{\max } \\
/ I C_{50} \\
\text { Meso }\end{array}$ & $\begin{array}{l}I_{50} \\
P M P \\
\mu M\end{array}$ & $\begin{array}{l}C_{\max } \\
/ I C_{50} \\
P M P\end{array}$ & $\begin{array}{l}\mathrm{IC}_{50} \\
\text { App } \\
\mu \mathrm{M}\end{array}$ & $\begin{array}{l}C_{\max } \\
/ I C_{50} \\
\text { App }\end{array}$ & $\begin{array}{c}I C_{50} \\
\text { Ova } \\
\mu \mathrm{M}\end{array}$ & $\begin{array}{l}C_{\max } \\
/ I C_{50} \\
\text { Ova }\end{array}$ \\
\hline \multicolumn{11}{|l|}{ Oxa [23] } \\
\hline $573 \mu \mathrm{M}^{\ddagger}$ & 31 & 18.5 & 12 & 47.7 & 29 & 19.9 & 35 & 16.4 & 15 & 38.2 \\
\hline \multicolumn{11}{|l|}{ Cis [24] } \\
\hline $92 \mu \mathrm{M}^{\ddagger}$ & 23 & 4.0 & 8 & 11.5 & 25 & 3.7 & 30 & 3.1 & 9 & 10.2 \\
\hline \multicolumn{11}{|l|}{ MMC [25] } \\
\hline $30 \mu \mathrm{M}^{\ddagger}$ & 20 & 1.5 & 5 & 6 & 16 & 1.9 & 19 & 1.6 & 8 & 3.7 \\
\hline \multicolumn{11}{|l|}{ Iri [17] } \\
\hline $197 \mu \mathrm{M}^{\ddagger}$ & 139 & 1.4 & 75 & 2.6 & 290 & 0.7 & 150 & 1.3 & 100 & 2.0 \\
\hline \multicolumn{11}{|l|}{ Dox [14] } \\
\hline $17 \mu M^{\ddagger}$ & 2.8 & 6.1 & 0.9 & 19.1 & 2.0 & 8.6 & 2.3 & 7.5 & 1.2 & 14.3 \\
\hline
\end{tabular}

${ }^{+}$Maximum concentration achieved at the beginning of the IPC treatment.

‡ Dosage during HIPEC: oxa $-460 \mathrm{mg} / \mathrm{m}^{2}$, cis $-50 \mathrm{mg} / \mathrm{m}^{2}, \mathrm{MMC}-35 \mathrm{mg} / \mathrm{m}^{2}$, Iri $-350 \mathrm{mg} / \mathrm{m}^{2}$, Dox $-15 \mathrm{mg} / \mathrm{m}^{2}$.

Abbreviations: IPC intraperitoneal chemotherapy, CRC colorectal cancer, Meso mesothelioma, PMP pseudomyxoma peritonei, App appendix cancer, Ova ovarian cancer, Oxa oxaliplatin, Cis cisplatin, MMC mitomycin C, Iri irinotecan, Dox doxorubicin, N/A not available, HIPEC hyperthermic intraperitoneal chemotherapy.

with similar chemotherapeutics seems appropriate. For IPC in PC from CRC and appendix cancer, there are two commonly used drugs, i.e. mitomycin $\mathrm{C}$ or oxaliplatin. The results of the current study do not give a clear answer to which drug is best but with mitomycin $C$ one may only reach intraperitoneal concentrations that approximate the tumor cell $\mathrm{IC}_{50}$ values whereas with oxaliplatin, one might reach 18 times greater a concentration ip. Thus, oxaliplatin seems to be a more suitable drug for IPC. Interestingly, oxaliplatin appears to have the best $\mathrm{C}_{\max } \mathrm{IP} / \mathrm{IC}_{50}$ ratio for the other PC diagnoses as well (Table 3).

The variability in ex vivo drug sensitivity in the CRC subgroup was large, as also observed in a previous study on tumor samples from PC [30], ranging from virtually no to total cell death within the concentration range tested. In principle, this argues, together with the observations of low cross-resistance in individual tumor samples, in favor of individualized choice of drug for IPC.

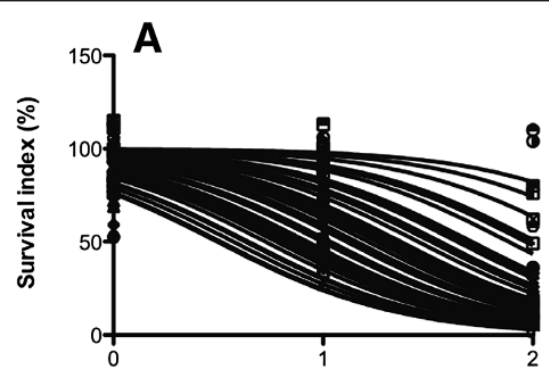

Log oxaliplatin concentration $(\mu \mathrm{M})$

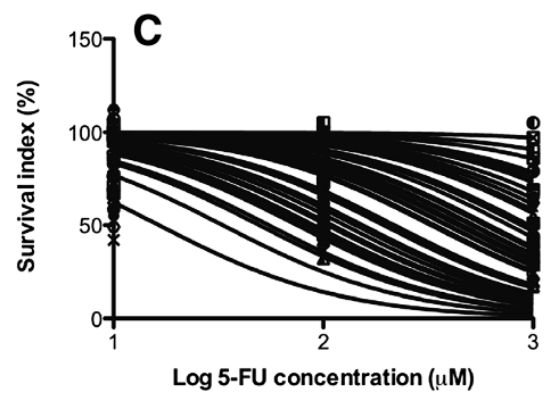

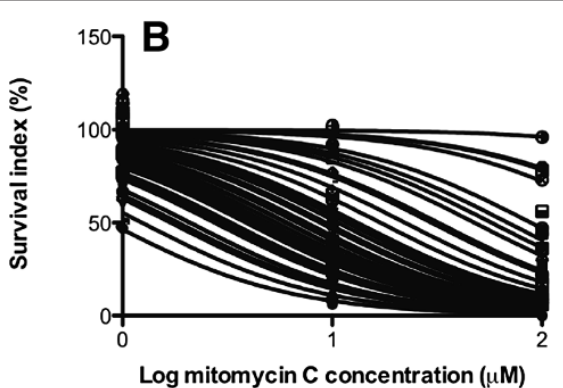

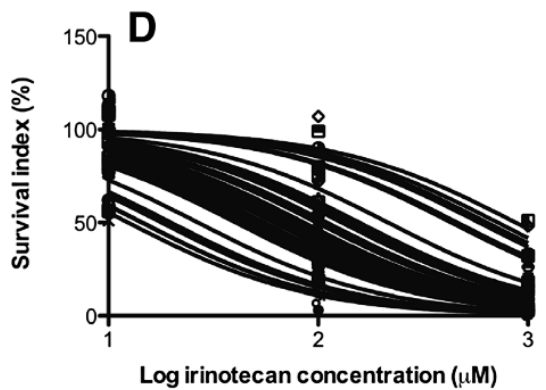

Figure 2 Tumor cell sensitivity, expressed as survival index (SI\%) of the colorectal cancer samples for the indicated standard cytotoxic drugs. The curves represent the non-linear regression lines calculated for all individual samples included. The dots are individual patient data points and illustrate together with the individual curves the great variability in drug sensitivity between individual samples. Panel $\mathbf{A}$ : oxaliplatin, B: mitomycin C, C: 5-fluorouracil, D: irinotecan. 


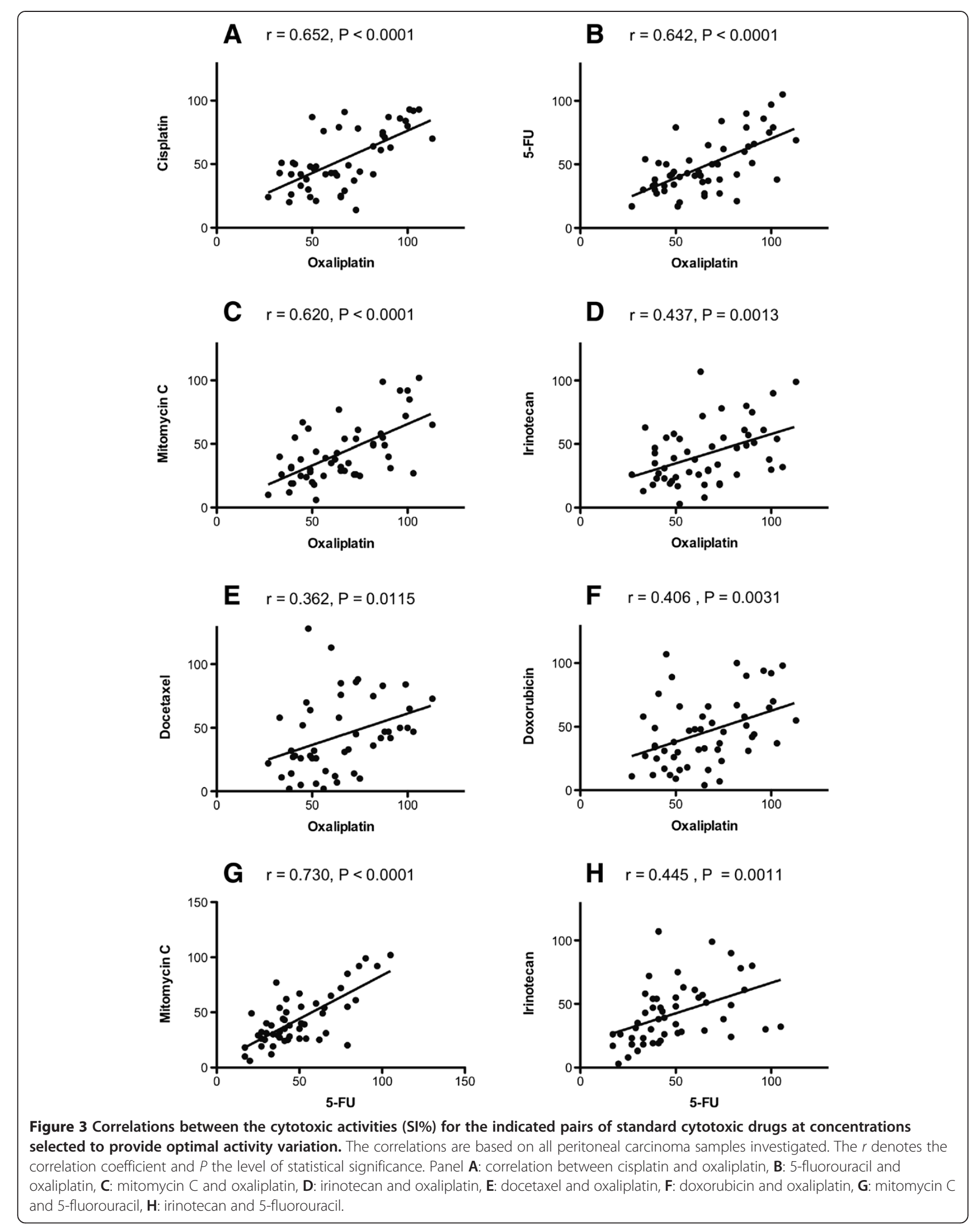


Table 4 Mean IC 50 values of drugs grouped according to histopathological categories and previous treatment in the CRC subgroup

\begin{tabular}{|c|c|c|c|c|c|c|c|c|c|c|c|}
\hline \multirow{3}{*}{$\begin{array}{l}\text { Drugs } \\
n=49+\end{array}$} & \multicolumn{2}{|c|}{ Prior Chemotherapy } & \multicolumn{2}{|c|}{ Lymph node } & \multicolumn{2}{|c|}{ Mucinous } & \multicolumn{3}{|c|}{ Tumour differentiation } & \multicolumn{2}{|c|}{ Signet cell } \\
\hline & Yes & No & Pos & Neg & Yes & No & Poor & Moderate & Well & Yes & No \\
\hline & $n=39$ & $n=10$ & $\mathrm{n}=37$ & $n=12$ & $n=29$ & $n=20$ & $n=19$ & $n=24$ & $n=6$ & $n=6$ & $n=43$ \\
\hline Oxa & 31.7 & $30.1(0.9)$ & 31.0 & $32.4(0.9)$ & 36.9 & $22.6(0.1)$ & 34.7 & $23.9(0.5)$ & 35.3 & 29.1 & $31.6(0.9)$ \\
\hline Cis & 21.6 & $26.2(0.6)$ & 20.1 & $23.8(0.9)$ & 29.7 & $13.6(0.05)$ & 23.3 & $17.0(0.4)$ & 32.8 & 13.7 & $24.7(0.4)$ \\
\hline Mel & 53.3 & $52.2(0.9)$ & 56.1 & $53.1(0.8)$ & 52.4 & $53.8(0.9)$ & 49.1 & $53.3(0.9)$ & 54.8 & 44.2 & $52.9(0.5)$ \\
\hline $5-\mathrm{FU}$ & 539.5 & $475.4(0.6)$ & 497.5 & $604.9(0.4)$ & 542.1 & $455.5(0.4)$ & 521.5 & $426.6(0.3)$ & 689.9 & 490.4 & $519.2(0.9)$ \\
\hline MMC & 19.1 & $22.5(0.7)$ & 23.3 & $12.0(0.3)$ & 24.6 & $14.2(0.2)$ & 12.8 & $20.5(0.4)$ & 29.4 & 8.68 & $21.2(0.3)$ \\
\hline Iri & 131.7 & $163.1(0.6)$ & 153.3 & $111.2(0.5)$ & 183.9 & $81.1(0.07)$ & 122.0 & $163.5(0.8)$ & 129.2 & 84.4 & $151.6(0.5)$ \\
\hline Doce & 15.0 & $21.5(0.3)$ & 18.5 & $13.8(0.4)$ & 17.2 & $16.6(0.9)$ & 16.9 & $17.9(0.8)$ & 12.4 & 23.5 & $14.4(0.1)$ \\
\hline Dox & 2.80 & $2.98(0.9)$ & 3.34 & $1.59(0.1)$ & 2.96 & $2.78(0.9)$ & 1.74 & $3.51(0.3)$ & 2.86 & 1.20 & $2.96(0.3)$ \\
\hline
\end{tabular}

Numbers in parentheses represent p-values from a Student t-test with the exception of tumor grading which was calculated with a one-way ANOVA.

${ }^{\dagger}$ Missing histopathological data in 3 patients.

Abbreviations: Pos positive, Neg negative, Oxa oxaliplatin, Cis cisplatin, 5-FU 5-fluorouracil, MMC mitomycin C, Iri irinotecan, Doce docetaxel, Dox doxorubicin.

This would require tumor tissue sampling and ex vivo drug sensitivity measurements prior to CRS and IPC. Although this would complicate the logistics, it would be feasible since tumor tissue is accessible by laparoscopy and the assay time is 3 days. However, well designed clinical trials evaluating clinical benefit from ex vivo drug sensitivity testing are unfortunately few, sub-optimally designed and do not allow firm conclusions [31].

In $\mathrm{PC}$ from $\mathrm{CRC}$, mucinous tumors were generally less drug sensitive than non-mucinous tumors. This is in line with previous results that mucinous CRC is less responsive to systemic chemotherapy than non-mucinous CRC [32]. Interestingly, oxaliplatin and 5-FU were essentially equally active in chemotherapy naïve and previously treated patients. This may be due to patient selection; i.e. previously treated patients suitable for CRS and IPC are those without drug resistant tumors. Still, there is no support for selection of drugs for IPC based on prior treatment status. The same conclusion holds for lymph node status, tumor grade and presence of signet ring cells.

Doxorubicin drug sensitivity was found to be an independent prognostic factor for TTP. This might well

Table 5 Univariate and multivariable Cox regression model for TTP according to dichotomised IC 50 drug sensitivity values (above or below the median value) and clinicopathological variables for the CRC subgroup

\begin{tabular}{|c|c|c|c|c|c|}
\hline$n=47$ & $\begin{array}{c}\text { Median TTP } \\
\text { Months }\end{array}$ & $\begin{array}{l}\text { Log-rank } \\
\qquad p\end{array}$ & $\begin{array}{c}\text { Univariate } \\
\text { HR }\end{array}$ & $\begin{array}{c}\text { Univariate } \\
p\end{array}$ & $\begin{array}{c}\text { Multivariate } \\
\qquad p\end{array}$ \\
\hline Treated vs. untreated & 10 vs. 5 & 0.06 & 0.5 & 0.2 & - \\
\hline Oxaliplatin ${ }^{+}$ & 5 vs. 5 & 0.8 & 0.9 & 0.9 & - \\
\hline Cisplatin $^{+}$ & 5 vs. 5 & 0.8 & 0.9 & 0.9 & - \\
\hline Melphalan $^{\dagger}$ & 12 vs. 7 & 0.8 & 1.1 & 0.8 & - \\
\hline $5-\mathrm{FU}^{+}$ & 6 vs. 5 & 0.7 & 0.9 & 0.7 & - \\
\hline Mitomycin $C^{+}$ & 12 vs. 4 & 0.2 & 0.6 & 0.2 & - \\
\hline Irinotecan ${ }^{\dagger}$ & 5 vs. 5 & 0.9 & 1.0 & 0.9 & - \\
\hline Docetaxel $^{+}$ & 10 vs. 4 & 0.6 & 0.8 & 0.6 & - \\
\hline Doxorubicin $^{+}$ & 12 vs. 1 & 0.008 & 0.4 & 0.009 & 0.02 \\
\hline Synch. vs. Metach. & 9 vs. 4 & 0.05 & 0.5 & 0.05 & 0.006 \\
\hline Vasc./neural vs. not & 2 vs. 10 & 0.05 & 2.3 & 0.03 & 0.12 \\
\hline Mucinous vs. not & 9 vs. 5 & 0.12 & 0.6 & 0.12 & - \\
\hline Lymph node + vs. not & 6 vs. 7 & 0.9 & 1.0 & 0.9 & - \\
\hline CCO vs. CC 1-3 & 18 vs. 4 & 0.002 & 0.3 & 0.001 & 0.002 \\
\hline
\end{tabular}

There was missing data concerning TTP in 5 patients.

${ }^{+}$Below vs. above the median $\mathrm{IC}_{50}$ value. Low $\mathrm{IC}_{50}$ indicates better sensitivity.

Abbreviations: TTP time to progression, CRC colorectal cancer, Synch synchronous, metach metachronous, vasc/neural vascular or neural invasion, CC completeness of cytoreduction. 


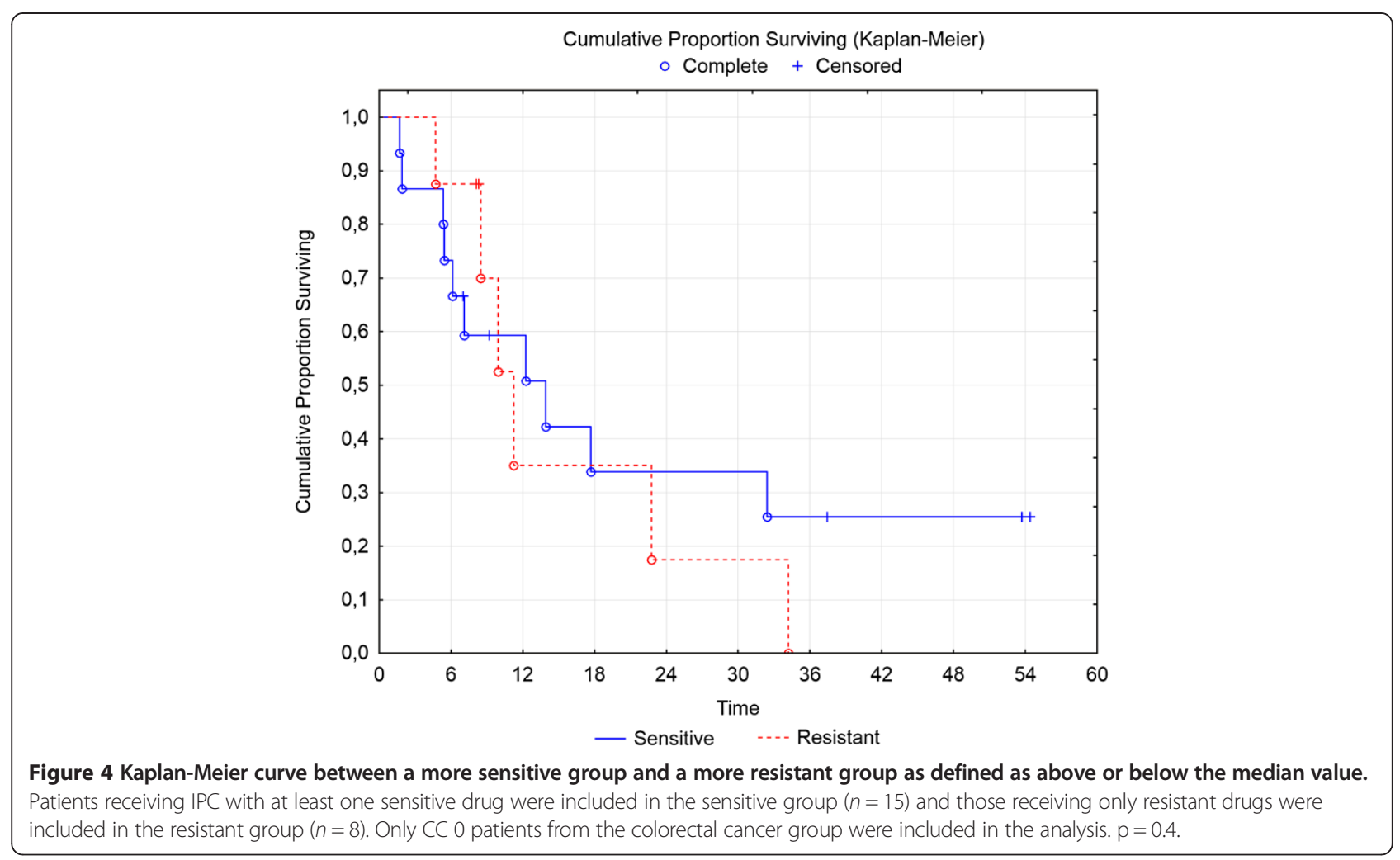

be a chance finding but one may speculate that doxorubicin drug sensitivity could indicate CRC tumor cell susceptibility to cancer drugs or that sensitivity to doxorubicin is associated with less aggressive tumor biology.

To support the conclusions and proposals above on the implications of our findings for the use of IPC for PC in the clinic and the role of ex vivo drug sensitivity testing, one would like to know, firstly, that IPC adds benefit to CRS and, secondly, that the ex vivo drug sensitivity data correlates to clinical outcome in patients undergoing IPC. Unfortunately, there is insufficient support for both of these aspects. Support for the benefit from the IPC when added to CRS only derives from one randomized trial in PC from gastric cancer [9], randomized trials in ovarian cancer [8], and from a rodent CRC PC model [11]. In PC from CRC, there was one randomized trial started to elucidate this issue but stopped prematurely after inclusion of 35 patients due to poor patient accrual [33]. There was no trend for benefit from IPC. There is, thus, need for a controlled trial to elucidate this important issue. One such study for CRC (Prodige 7) in France has soon completed its recruitment process.

In the CRC subset, the analysis of the relationship between ex vivo activity of the drugs given to the patient and the clinical outcome in terms of TTP showed no difference between 'sensitive' and 'resistant' patients in the short term; but unfortunately, the analysis suffers from major methodological problems related to the limited and heterogeneous data available making the results essentially inconclusive. The number of analyzable patients was a mere 23 ( 15 vs. 8, Figure 4 ) as the remaining patients were either open-and-close patients or did not reach complete cytoreduction during the surgery. Furthermore, heterogeneity of drug regimens administered compounded the problem of predictive analysis. A greater number of patients with sufficient follow-up are needed to make a reasonable ex vivo - clinical outcome analysis, which is of importance considering the possible long term effect indicated in Figure 4.

On the other hand, the lack of relationship between the ex vivo drug sensitivity and clinical outcome might be true. This could be the case if the FMCA does not report clinically relevant information. The assay differs from the in vivo situation in several aspects related to both pharmacokinetics and pharmacodynamics. Also relevant, is the dwell time that is significantly longer than in the IPC situation. This may have implications for cell-cycle dependant drugs. However, these differences are more or less inherent in all disease and treatment models, and this will reasonably mean that an ex vivo test like the FMCA will never show a perfect correlation to the clinical outcome. Other reasons for the possible lack of relationship between drug sensitivity and clinical outcome might be that IPC adds no benefit at all to CRS or, alternatively, that the drug concentrations reached during IPC are sufficiently high to overcome drug resistance. Taken together, 
there are a number of important issues left to investigate when it comes to adding IPC to CRS for treatment of PC.

\section{Conclusions}

Drug-sensitivity varies considerably between PC diagnoses and individual patients arguing for individualized therapy in IPC rather than standard diagnosis-specific therapy. However, in the current paradigm of treatment according to diagnosis, oxaliplatin is seemingly the preferred drug for IPC from a drug sensitivity and concentration perspective. In the CRC subset, analysis of correlation between ex vivo drug sensitivity and TTP was inconclusive due to the heterogeneous nature of the data.

\begin{abstract}
Abbreviations
IPC: Intraperitoneal chemotherapy; CRS: Cytoreductive surgery; PC: Peritoneal carcinomatosis; PMP: Pseudomyxoma peritonei; CRC: Colorectal cancer; 5-FU: 5-fluorouracil; TTP: Time to progression; HIPEC: Hyperthermic intraperitoneal chemotherapy; MNCs: Mononuclear cells; SPIC: Sequential postoperative intraperitoneal chemotherapy; FMCA: Fluorometric microculture cytotoxicity assay; FDA: Fluorescein diacetate; CC: Completeness of cytoreduction; IC50: 50\% inhibitory concentration.
\end{abstract}

\section{Competing interests}

We, the authors, have no financial or non-financial competing interests.

\section{Authors' contributions}

PC, HM, WG and PN participated in the study concept and design and in data collection or provision of subject material. $\mathrm{HK}, \mathrm{RL}$, and PN contributed in the area of chemosensitivity testing. All authors participated in the data analysis/interpretation as well as in the article writing/critiquing and have given approval to the final draft.

\section{Acknowledgements}

The skilful technical assistance of Anna-Karin Lannergård, Kristin Blom and Annika Jonasson for tumor cell preparation and ex vivo drug sensitivity testing is gratefully acknowledged. This study was supported by grants from the Swedish Cancer Society, the Swedish Foundation for Strategic Research and Lions Cancer Research Fund.

\section{Author details}

${ }^{1}$ Department of Surgical Sciences, Section of Surgery, Akademiska Sjukhuset, Uppsala University, Uppsala S-751 85, Sweden. ${ }^{2}$ Department of Medical Sciences, Section of Clinical Pharmacology, Akademiska Sjukhuset, Uppsala University, Uppsala S-751 85, Sweden. ${ }^{3}$ Department of Radiology, Oncology, and Radiation Sciences, Section of Oncology, Akademiska Sjukhuset, Uppsala University, Uppsala S-751 85, Sweden.

Received: 2 November 2012 Accepted: 13 September 2013 Published: 24 September 2013

\section{References}

1. Glehen $O$, Kwiatkowski F, Sugarbaker PH, Elias D, Levine EA, De Simone M, Barone R, Yonemura Y, Cavaliere F, Quenet F, Gutman M, Tentes AAK, Lorimier G, Bernard JL, Bereder JM, Porcheron J, Gomez-Portilla A, Shen P, Deraco M, Rat P: Cytoreductive surgery combined with perioperative intraperitoneal chemotherapy for the management of peritoneal carcinomatosis from colorectal cancer: a multi-institutional study. J Clin Oncol 2004, 22:3284-3292.

2. Elias D, Gilly F, Boutitie F, Quenet F, Bereder J-M, Mansvelt B, Lorimier G, Dubè $P$, Glehen O: Peritoneal Colorectal Carcinomatosis Treated With Surgery and Perioperative Intraperitoneal Chemotherapy: Retrospective Analysis of 523 Patients From a Multicentric French Study. J Clin Oncol 2010, 28:63-68.

3. Cavaliere F, De Simone M, Virzi S, Deraco M, Rossi CR, Garofalo A, Di Filippo F, Giannarelli D, Vaira M, Valle M, Pilati P, Perri P, La Pinta M, Monsellato I, Guadagni F: Prognostic factors and oncologic outcome in 146 patients with colorectal peritoneal carcinomatosis treated with cytoreductive surgery combined with hyperthermic intraperitoneal chemotherapy: Italian multicenter study S.I.T.I.L.O. Eur J Surg Oncol 2011, 37:148-154.

4. Verwaal VJ, van Ruth S, Witkamp A, Boot H, van Slooten G, Zoetmulder FAN: Long-term survival of peritoneal carcinomatosis of colorectal origin. Ann Surg Oncol 2005, 12:65-71.

5. Cashin PH, Graf W, Nygren P, Mahteme H: Intraoperative hyperthermic versus postoperative normothermic intraperitoneal chemotherapy for colonic peritoneal carcinomatosis: a case-control study. Ann Oncol 2012, 23:647-652.

6. Benoit L, Duvillard C, Rat P, Chauffert B: The effect of intra-abdominal temperature on the tissue and tumor diffusion of intraperitoneal cisplatin in a model of peritoneal carcinomatosis in rats. Chirurgie 1999, 124:375-379.

7. Sommariva A, Pilati P, Rossi CR: Cyto-reductive Surgery combined with Hyperthermic Intra-peritoneal Chemotherapy for Peritoneal Surface Malignancies: current treatment and results. Cancer Treat Rev 2012, 38:258-268.

8. Armstrong DK, Bundy B, Wenzel L, Huang HQ, Baergen R, Lele S, Copeland $\sqcup$, Walker JL, Burger RA: Intraperitoneal cisplatin and paclitaxel in ovarian cancer. N Engl J Med 2006, 354:34-43.

9. Yang X-J, Huang C-Q, Suo T, Mei L-J, Yang G-L, Cheng F-L, Zhou Y-F, Xiong $B$, Yonemura $Y$, Li Y: Cytoreductive surgery and hyperthermic intraperitoneal chemotherapy improves survival of patients with peritoneal carcinomatosis from gastric cancer: final results of a phase III randomized clinical trial. Ann Surg Oncol 2011, 18:1575-1581.

10. Verwaal VJ, van Ruth $S$, de Bree E, van Sloothen GW, van Tinteren H, Boot $H$, Zoetmulder FAN: Randomized trial of cytoreduction and hyperthermic intraperitoneal chemotherapy versus systemic chemotherapy and palliative surgery in patients with peritoneal carcinomatosis of colorectal cancer. J Clin Oncol 2003, 21:3737-3743.

11. Klaver YLB, Hendriks T, Lomme RMLM, Rutten HJT, Bleichrodt RP, de Hingh IHJT: Hyperthermia and intraperitoneal chemotherapy for the treatment of peritoneal carcinomatosis: an experimental study. Ann Surg 2011, 254:125-130.

12. Dedrick RL: Theoretical and experimental bases of intraperitoneal chemotherapy. Semin Oncol 1985, 12:1-6.

13. Ceelen WP, Påhlman L, Mahteme H: Pharmacodynamic aspects of intraperitoneal cytotoxic therapy. Cancer Treat Res 2007, 134:195-214.

14. Speeten K, Stuart OA, Mahteme H, Sugarbaker PH: A pharmacologic analysis of intraoperative intracavitary cancer chemotherapy with doxorubicin. Cancer Chemother Pharmacol 2008, 63:799-805.

15. Cotte E, Colomban O, Guitton J, Tranchand B, Bakrin N, Gilly F-N, Glehen O, Tod M: Population pharmacokinetics and pharmacodynamics of cisplatinum during hyperthermic intraperitoneal chemotherapy using a closed abdominal procedure. J Clin Pharmacol 2011, 51:9-18.

16. Elias D, Bonnay M, Puizillou JM, Antoun S, Demirdjian S, El OA, Pignon JP, Drouard-Troalen L, Ouellet JF, Ducreux M: Heated intra-operative intraperitoneal oxaliplatin after complete resection of peritoneal carcinomatosis: pharmacokinetics and tissue distribution. Ann Oncol 2002, 13:267-272.

17. Elias D, Matsuhisa T, Sideris L, Liberale G, Drouard-Troalen L, Raynard B, Pocard M, Puizillou JM, Billard V, Bourget P, Ducreux M: Heated intra-operative intraperitoneal oxaliplatin plus irinotecan after complete resection of peritoneal carcinomatosis: pharmacokinetics, tissue distribution and tolerance. Ann Oncol 2004, 15:1558-1565.

18. Jacquet $P$, Averbach A, Stephens AD, Stuart OA, Chang D, Sugarbaker $\mathrm{PH}$ : Heated intraoperative intraperitoneal mitomycin $\mathrm{C}$ and early postoperative intraperitoneal 5-fluorouracil: pharmacokinetic studies. Oncol 1998, 55:130-138.

19. Csóka K, Tholander B, Gerdin E, de la Torre M, Larsson R, Nygren P: In vitro determination of cytotoxic drug response in ovarian carcinoma using the fluorometric microculture cytotoxicity assay (FMCA). Int J Cancer 1997, 72:1008-1012.

20. Frost B-M, Nygren P, Gustafsson G, Forestier E, Jonsson OG, Kanerva J, Nygaard $R$, Schmiegelow $K$, Larsson $R$, Lönnerholm G: Increased in vitro cellular drug resistance is related to poor outcome in high-risk childhood acute lymphoblastic leukemia. Br J Haematol 2003, 122:376-385.

21. Jonsson E, Dhar S, Jonsson B, Nygren P, Graf W, Larsson R: Differential activity of topotecan, irinotecan and SN-38 in fresh human tumour cells but not in cell lines. Eur J Cancer 2000, 36:2120-2127.

22. Lindhagen $E$, Nygren $P$, Larsson R: The fluorometric microculture cytotoxicity assay. Nat Protoc 2008, 3:1364-1369.

23. Mahteme H, Wallin I, Glimelius B, Påhlman L, Ehrsson H: Systemic exposure of the parent drug oxaliplatin during hyperthermic intraperitoneal perfusion. Eur J Clin Pharmacol 2008, 64:907-911. 
24. Cashin PH, Ehrsson H, Wallin I, Nygren P, Mahteme H: Pharmacokinetics of cisplatin during hyperthermic intraperitoneal treatment of peritoneal carcinomatosis. Eur J Clin Pharmacol 2012: . DOl: 10.1007/s00228-012-1405-4.

25. van Ruth S, Verwaal VJ, Zoetmulder FA: Pharmacokinetics of intraperitoneal mitomycin C. Surg Oncol Clin N Am 2003, 12:771-780.

26. Nygren P, Fridborg H, Csoka K, Sundström C, de la Torre M, Kristensen J, Bergh J, Hagberg H, Glimelius B, Rastad J: Detection of tumor-specific cytotoxic drug activity in vitro using the fluorometric microculture cytotoxicity assay and primary cultures of tumor cells from patients. Int J Cancer 1994, 56:715-720.

27. Yan $T$, Welch $L$, Black $D$, Sugarbaker P: A systematic review on the efficacy of cytoreductive surgery combined with perioperative intraperitoneal chemotherapy for diffuse malignancy peritoneal mesothelioma. Ann Oncol 2007, 18:827-834.

28. Glehen O, Gilly FN, Boutitie F, Bereder JM, Quenet F, Sideris L, Mansvelt B, Lorimier G, Msika S, Elias D: Toward curative treatment of peritoneal carcinomatosis from nonovarian origin by cytoreductive surgery combined with perioperative intraperitoneal chemotherapy: a multi-institutional study of 1,290 patients. Cancer 2010, 116:5608-5618.

29. Yan TD, Black D, Savady R, Sugarbaker PH: A systematic review on the efficacy of cytoreductive surgery and perioperative intraperitoneal chemotherapy for pseudomyxoma peritonei. Ann Surg Oncol 2007, 14:484-492.

30. Mahteme H, von Heideman A, Grundmark B, Tholander B, Påhlman L, Glimelius B, Larsson R, Graf W, Nygren P: Heterogeneous activity of cytotoxic drugs in patient samples of peritoneal carcinomatosis. Eur J Surg Oncol 2008, 34:547-552.

31. Cortazar P, Johnson BE: Review of the Efficacy of Individualized Chemotherapy Selected by In Vitro Drug Sensitivity Testing for Patients with Cancer. J Clin Oncol 1999, 17:1625-1625.

32. Mekenkamp LIM, Heesterbeek KJ, Koopman M, Tol J, Teerenstra S, Venderbosch S, Punt CJA, Nagtegaal ID: Mucinous adenocarcinomas: poor prognosis in metastatic colorectal cancer. Eur J Cancer 2012, 48:501-509.

33. Elias D, Delperro J-R, Sideris L, Benhamou E, Pocard M, Baton O, Giovannini $M$, Lasser P: Treatment of peritoneal carcinomatosis from colorectal cancer: impact of complete cytoreductive surgery and difficulties in conducting randomized trials. Ann Surg Oncol 2004, 11:518-521.

doi:10.1186/1471-2407-13-435

Cite this article as: Cashin et al:: Activity ex vivo of cytotoxic drugs in patient samples of peritoneal carcinomatosis with special focus on colorectal cancer. BMC Cancer 2013 13:435.

\section{Submit your next manuscript to BioMed Central and take full advantage of:}

- Convenient online submission

- Thorough peer review

- No space constraints or color figure charges

- Immediate publication on acceptance

- Inclusion in PubMed, CAS, Scopus and Google Scholar

- Research which is freely available for redistribution 